\title{
Prevalence and Psychiatric Comorbidities of Internet Addiction in a Nationwide Sample of Korean Adults
}

\author{
Subin Park ${ }^{1}$, Hong Jin Jeon ${ }^{2}$, Jae Nam Bae ${ }^{3}$, Su Jeong Seong ${ }^{4}$, and Jin Pyo Hong ${ }^{2} \bowtie$ \\ ${ }^{1}$ Department of Research Planning, Mental Health Research Institute, National Center for Mental Health, Seoul, Republic of Korea \\ ${ }^{2}$ Department of Psychiatry, Samsung Medical Center, Sungkyunkwan University School of Medicine, Seoul, Republic of Korea \\ ${ }^{3}$ Department of Psychiatry, Inha University of College of Medicine, Incheon, Republic of Korea \\ ${ }^{4}$ Department of Psychiatry, Hallym University Medical Center, Seoul, Republic of Korea
}

The goal of the study was to examine the prevalence and psychiatric comorbidities of Internet addiction in a nationwide sample of Korean adults. Among a total of 6,022 participants involved in Korean Epidemiological Catchment Area study in 2011, 3,723 Internet users were assessed for their Internet addiction using Young's 8-item Diagnostic Questionnaire. The results showed that of the 3,723 Internet users, 39 (1.0\%) had Internet addiction. Addicted Internet users were younger, more likely to be males and unemployed compared to non-addicted Internet users. Internet addiction was associated with alcohol and nicotine use disorders, depressive disorders, and anxiety disorder after controlling for age, gender, and occupational status. Internet addiction was associated with suicidal plans even after controlling for psychiatric disorders and socio-demographic factors. This study showed that Internet addiction is highly associated with variety of different psychiatric disorders and suicidal plans. Therefore, clinicians should carefully evaluate them when treating addicted Internet users.

Psychiatry Investig 2017;14(6):879-882

Key Words Internet addiction, Mental disorders, Suicide, Korea.

\section{INTRODUCTION}

Internet addiction (IA) is characterized by inability to control Internet use which interferes with one's daily life. ${ }^{1}$ IA is not listed in the fifth edition of the Diagnostic and Statistical Manual of Mental Disorders (DSM-5). However, Internet Gaming Disorder is listed in Section III as a condition warranting more scientific research and clinical experience before it might be considered as a formal disorder. ${ }^{2}$ IA broadly includes other types of addictive internet use (i.e., addictive use of social networking sites, addictive online shopping, and excessive internet information retrieval) and internet gaming. ${ }^{3}$

The prevalence of IA varies among populations. It ranges from $0.3 \%{ }^{4}$ to $37 \%{ }^{5}$ This variability across studies might have

Received: November 2, 2016 Revised: February 14, 2017

Accepted: March 4, 2017 Available online: September 29, 2017

$\triangle$ Correspondence: Jin Pyo Hong, MD, PhD

Department of Psychiatry, Samsung Medical Center, Sungkyunkwan University School of Medicine, 81 Irwon-ro, Gangnam-gu, Seoul 06351, Republic of Korea

Tel: +82-2-3410-3585, Fax: +82-2-3410-0050, E-mail: suhurhong@gmail.com (c) This is an Open Access article distributed under the terms of the Creative Commons Attribution Non-Commercial License (http://creativecommons.org/licenses/bync/4.0) which permits unrestricted non-commercial use, distribution, and reproduction in any medium, provided the original work is properly cited. resulted from variations in Internet accessibility in different countries, definitions of IA, assessment tools, and age ranges of different population. ${ }^{6}$ Significant association between IA and psychiatric comorbidity has been documented in numerous studies (for a review ${ }^{3,5,7}$ ). However, most studies have been focused on groups of students or adolescents in school-based studies or Internet users in online surveys. Ho et al. ${ }^{3}$ have conducted a meta-analysis of the literature and determined the association between IA and psychiatric comorbidity including 8 studies comprising 1,641 patients with IA and 11,210 controls. Positive associations between IA and alcohol abuse, attention-deficit hyperactivity disorder, depression, and anxiety have been found, with pooled prevalence rates ranging from $13.5 \%$ for alcohol abuse to $26.3 \%$ for depression. ${ }^{3}$ To the best of our knowledge, there are only two published studies on the prevalence and comorbidities of IA in a random sample of the general population. Aboujaoude et $\mathrm{al}^{4}{ }^{4}$ have reported a prevalence of problematic Internet use of $0.3 \%$ in a general population of the United States ( $\geq 18$ years of age, $n=2,513$ ). Bakken et al. ${ }^{8}$ have reported a prevalence of IA of $1.0 \%$ in the general Norwegian population (16-74 years, $n=3,399)$ with a linear relationship between the prevalence of self-reported mental health problems and increasing Internet-related problems. 
Given the paucity of using general population of random adults sample to study IA, we investigated the prevalence and psychiatric comorbidities of IA in a nationally representative sample of adults aged $18^{-} 70$ years in South Korea.

\section{METHODS}

\section{Sample}

We examined data from the Korean Epidemiologic Catchment Area (KECA) study conducted in 2011. ${ }^{9}$ The objective of KECA was to evaluate the lifetime and 12-month prevalence, demographic and socioeconomic factors, and comorbidities of psychiatric disorders for the Diagnostic and Statistical Manual of Mental Disorders, Fourth Edition (DSM-IV) of Korean adults. ${ }^{9-11}$ KECA selected subjects using a stratified, multi-stage, and clustered sample design in reference to a population census conducted by community registry offices in 2010. ${ }^{9}$ The individual whose birthday was the earliest was chosen for every selected household based on the day of the birth month. After excluding 1,628 individuals who refused to participate, 6,022 subjects completed the diagnostic interview. ${ }^{9}$ For these participants, Internet use was initially assessed using the following dichotomous question: "Have you ever used Internet in the past 1 month?" A total of 3,723 respondents who did used Internet in the past 1 month were assessed for IA using Young's Diagnostic Questionnaire (DQ). All procedures of the study protocol were approved by the Institutional Review Board of Seoul National University College of Medicine (IRB No. C-1104-092-359). All participants were fully informed of the study objectives and written consent was obtained from all participants.

\section{Measurement}

IA was assessed with Young's 8-item Diagnostic Questionnaire (DQ). ${ }^{12}$ In the $\mathrm{DQ}$, respondents who answered "yes" to five or more criteria out of eight were classified as addicted Internet users. The others were classified as usual Internet users. Participants were also asked to choose from additional choices of social media, Internet gaming, Internet retrieval, use of e-mail, videos, pornography, online shopping, and others as main area of Internet use. Psychiatric disorders were measured with the Korean version of the Composite International Diagnostic Interview (K-CIDI), ${ }^{12,13}$ a fully structured diagnostic modality designed to determine psychiatric diagnoses based on DSM-IV. ${ }^{14}$ The inter-rater reliability, test/retest reliability, and validity of the K-CIDI ranged from $0.86-1.00,0.42-0.89$, and $0.50-1.00$ in kappa value, respectively.

\section{Statistical analysis}

Weighted values for each respondent were applied to esti- mate the national population in respect of age and gender for each catchment area using the 2010 Korean National Statistical Office census. ${ }^{9}$ First, we performed Pearson's chi-square test to compare gender, age group, economic and occupational status, residential region, and comorbid psychiatric disorders between usual groups. We also performed multivariate logistic regression analyses to estimate the odds ratios (ORs) and 95\% confidence intervals (CIs) of lifetime psychiatric disorders after controlling for socio-demographic variables. SPSS (version 21.0; IBM Corp., Armonk, NY, USA) was used for all statistical analyses. Statistical significance was considered when pvalue was less than 0.05 .

\section{RESULTS}

Of the total of 3,723 participants, 39 (1.0\%, 95\% CI: $0.7-1.3 \%)$ were classified as addicted Internet users. Regarding the main area of Internet use, addictive Internet users were more likely to use social media ( $20.5 \%$ vs. $\left.8.8 \%, \chi^{2}=4.89, \mathrm{p}=0.027\right)$ and Internet game ( $33.3 \%$ vs. $\left.12.2 \%, \chi^{2}=15.81, \mathrm{p}<0.001\right)$ compared to usual Internet users. They were less likely to use Internet for information retrieval ( $34.2 \%$ vs. $56.2 \%, \chi^{2}=7.41, \mathrm{p}=0.006$ ) compared to usual Internet users.

Addictive Internet users were younger $(\mathrm{p}=0.009)$ than usual Internet users. They were more likely to be male $(\mathrm{p}=0.027)$ and unemployed $(\mathrm{p}=0.006)$ compared to usual Internet users. Educational status and economic status were not significantly different between the two groups. A total of $74.4 \%$ of addicted Internet users and $29.1 \%$ of usual Internet users had at least one DSM-IV psychiatric disorder (Table 1).

IA was positively associated with a wide range of psychiatric comorbidities, including alcohol [adjusted odd ratio $(\mathrm{AOR})=$ $2.10,95 \% \mathrm{CI}=1.02-4.29, \mathrm{p}=0.043]$ and nicotine use disorders (AOR $=3.98,95 \% \mathrm{CI}=1.82-8.69, \mathrm{p}=0.001$ ), depressive disorders ( $\mathrm{AOR}=9.67,95 \% \mathrm{CI}=4.86-19.23, \mathrm{p}<0.001)$, anxiety disorder (AOR=8.17, 95\% CI $=4.14-16.09, \mathrm{p}<0.001)$, and any DSM-IV psychiatric disorder $(\mathrm{AOR}=6.94,95 \% \mathrm{CI}=3.32-14.55, \mathrm{p}<0.001)$ after controlling for age, gender, and occupational status. When anxiety disorder was classified into several more specific types, IA was significantly and positively associated with generalized anxiety disorder $(\mathrm{AOR}=15.39,95 \% \mathrm{CI}=6.16-38.43, \mathrm{p}<0.001)$, specific phobia $(\mathrm{AOR}=2.76,95 \% \mathrm{CI}=1.03-7.36, \mathrm{p}=0.043)$, social phobia $(\mathrm{AOR}=7.02,95 \% \mathrm{CI}=1.34-36.88, \mathrm{p}=0.021)$, and post-traumatic stress disorder ( $\mathrm{AOR}=8.32,95 \% \mathrm{CI}=2.37-29.25$, $\mathrm{p}=0.001)$.

\section{DISCUSSION}

The present study provided the first representative estimated prevalence of IA in a general population of Korean adults. Our 
Table 1. Socio-demographic and clinical characteristics of addicted Internet users and usual Internet users

\begin{tabular}{|c|c|c|c|c|}
\hline & Usual Internet users $(\mathrm{N}=3686)$ & Addicted Internet users $(\mathrm{N}=39)$ & \multirow{2}{*}{$\chi^{2}$} & \multirow[b]{2}{*}{$\mathrm{p}$} \\
\hline & $\%$ & $\%$ & & \\
\hline Age (years) & & & 9.48 & 0.009 \\
\hline $18-34$ & 47.2 & 71.8 & & \\
\hline $35-54$ & 45.7 & 23.1 & & \\
\hline 55 or older & 7.1 & 5.1 & & \\
\hline Gender & & & 4.89 & 0.027 \\
\hline Male & 54.1 & 71.8 & & \\
\hline Female & 45.9 & 28.2 & & \\
\hline Education & & & 0.19 & 0.664 \\
\hline Less than high school & 40.2 & 43.6 & & \\
\hline High school or more & 59.8 & 56.4 & & \\
\hline Occupation & & & 7.61 & 0.006 \\
\hline Employed & 56.5 & 34.2 & & \\
\hline Unemployed & 43.5 & 65.8 & & \\
\hline Residence & & & 0.001 & 0.977 \\
\hline Urban & 81.8 & 81.6 & & \\
\hline Rural & 18.2 & 18.4 & & \\
\hline Family income & & & 0.69 & 0.407 \\
\hline$\leq 1000 \$$ & 27.3 & 34.6 & & \\
\hline$>1000 \$$ & 72.7 & 65.4 & & \\
\hline \multicolumn{5}{|l|}{ Psychiatric disorders } \\
\hline Alcohol use disorder & 15.5 & 28.9 & 5.11 & 0.024 \\
\hline Nicotine use disorder & 7.6 & 25.6 & 17.50 & $<0.001$ \\
\hline Depressive disorder & 6.2 & 36.8 & 58.44 & $<0.001$ \\
\hline Any anxiety disorder & 8.3 & 39.5 & 46.02 & $<0.001$ \\
\hline Generalized anxiety disorder & 1.5 & 18.4 & 65.32 & $<0.001$ \\
\hline Specific phobia & 5.5 & 13.5 & 4.48 & 0.034 \\
\hline Social phobia & 0.5 & 5.1 & 13.74 & $<0.001$ \\
\hline Obsessive-compulsive disorder & 0.7 & 7.7 & 23.37 & 0.004 \\
\hline Post-traumatic stress disorder & 1.3 & 7.7 & 12.00 & 0.015 \\
\hline Any DSM-IV psychiatric disorder & 29.1 & 74.4 & 37.92 & $<0.001$ \\
\hline
\end{tabular}

DSM-IV: Diagnostic and Statistical Manual of Mental Disorders, Fourth Edition

results revealed significant association between IA and other psychiatric disorders such as alcohol and nicotine use disorder, depressive disorder, and anxiety disorder.

Our findings of a $1.0 \%$ prevalence rate of IA among Korean internet users was similar to prevalence rate reported by a previous study conducted in Norway (1.0\%), but higher than that estimated in a previous study conducted in the United States $(0.3 \%)$. While more percentage of usual users used Internet retrieval to get useful information, addictive users were more likely to use social media and Internet gaming, consistent with the finding of Bakken et al. ${ }^{8}$ Addictive nature of Internet gaming has been previously recognized. In fact, Inter- net gaming disorder has been listed in DSM-5 as a condition for further research. ${ }^{2}$ In addition, addictive qualities of social networking sites have been recently addressed. ${ }^{15,16}$ They also require clinical attention.

Consistent with prior findings, ${ }^{8}$ addicted Internet users were more likely to be younger adults than usual users. This might be attributed to the fact that younger adults have easier access to technology. The finding of higher prevalence of IA in males is similar to that reported in prior studies. ${ }^{7,17,18}$ In addition, addicted Internet users were more likely to be unemployed, compatible with a positive association between IA and unsatisfactory financial status reported in a previous study. ${ }^{8}$ Exces- 
sive Internet use might provide a psychological escape from personal difficult situations such as unemployment. However, it might may further impede their social functioning as well. ${ }^{19}$

Our findings support the well-established associations between Internet addiction problem and a wide range of psychiatric disorders. ${ }^{3,5,7,8}$ As found in prior studies among clinical sample and adolescents as well as young adult population, IA was associated with increased prevalence of substance use disorder, ${ }^{5,8,18}$ depressive disorder, ${ }^{3,7,8,17,20}$ various types of anxiety disorder, ${ }^{3,7}$ and obsessive-compulsive disorder. ${ }^{5,78}$

This cross-sectional study has several limitations. First, it cannot determine the causal relationship between IA and comorbid psychiatric disorders. In addition, IA was assessed via participants' self-reporting. Thus, further prospective studies using more structured clinical assessments for IA are needed to classify IA as a predictive factor or consequence of other psychiatric disorders. Despite such limitations, our results indicated that IA was closely linked with diverse psychiatric disorders in a general adult population in Korea.

\section{Acknowledgments}

This study was funded by the Ministry of Health and Welfare, Republic of Korea and Intramural Research Grant from the National Center for Mental Health, Ministry of Health \& Welfare, Republic of Korea.

We thank 12 local investigators and 79 interviewers who helped this study.

\section{REFERENCES}

1. Mitchell P. Internet addiction: genuine diagnosis or not? Lancet 2000;355: 632.

2. American Psychiatric Association. Diagnostic and Statistical Manual of Mental Disorders DSM-5, 5th Ed. Washington: American Psychiatric Publishing; 2013.

3. Ho RC, Zhang MW, Tsang TY, Toh AH, Pan F, Lu Y, et al. The association between internet addiction and psychiatric co-morbidity: a metaanalysis. BMC Psychiatry 2014;14:183.

4. Aboujaoude E, Koran LM, Gamel N, Large MD, Serpe RT. Potential markers for problematic internet use: a telephone survey of 2,513 adults. CNS Spectr 2006;11:750-755.

5. Ko CH, Yen JY, Yen CF, Chen CS, Chen CC. The association between Internet addiction and psychiatric disorder: a review of the literature. Eur Psychiatry 2012;27:1-8.
6. Weinstein A, Lejoyeux M. Internet addiction or excessive internet use. Am J Drug Alcohol Abuse 2010;36:277-283.

7. Carli V, Durkee T, Wasserman D, Hadlaczky G, Despalins R, Kramarz $\mathrm{E}$, et al. The association between pathological internet use and comorbid psychopathology: a systematic review. Psychopathology 2013;46:1-13.

8. Bakken IJ, Wenzel HG, Gotestam KG, Johansson A, Oren A. Internet addiction among Norwegian adults: a stratified probability sample study. Scand J Psychol 2009;50:121-127.

9. Cho MJ, Seong SJ, Park JE, Chung IW, Lee YM, Bae A, et al. Prevalence and correlates of DSM-IV mental disorders in South Korean adults: the Korean epidemiologic catchment area study 2011. Psychiatry Investig 2015;12:164-170.

10. Cho MJ, Kim JK, Jeon HJ, Suh T, Chung IW, Hong JP, et al. Lifetime and 12-month prevalence of DSM-IV psychiatric disorders among Korean adults. J Nerv Ment Dis 2007;195:203-210.

11. Cho MJ, Chang SM, Lee YM, Bae A, Ahn JH, Son J, et al. Prevalence of DSM-IV major mental disorders among Korean adults: a 2006 National Epidemiologic Survey (KECA-R). Asian J Psychiatr 2010;3:26-30.

12. Cho MJ, Hahm BJ, Suh DW, Hong JP, Bae JN, Kim JK, et al. Development of a Korean version of the Composite International Diagnostic Interview (K-CIDI). J Korean Neuropsychiatr Assoc 2002;41:123-137.

13. World Health Organization: Composite International Diagnostic Interview (CIDI), Version 1.0. Geneva: World Health Organization; 1990.

14. Amerian Psychiatric Association. Diagnostic and Statistical Mannual of Mental Disorders, 4th, Text Revision ed. Washington, DC: Amerian Psychiatric Association; 2000.

15. Schou Andreassen C, Billieux J, Griffiths MD, Kuss DJ, Demetrovics Z, Mazzoni E, et al. The relationship between addictive use of social media and video games and symptoms of psychiatric disorders: a large-scale cross-sectional study. Psychol Addict Behav 2016;30:252-262.

16. Kuss DJ, Griffiths MD. Online social networking and addiction-a review of the psychological literature. Int J Environ Res Public Health 2011;8:3528-3552.

17. Yen JY, Ko CH, Yen CF, Wu HY, Yang MJ. The comorbid psychiatric symptoms of internet addiction: Attention Deficit and Hyperactivity Disorder (ADHD), depression, social phobia, and hostility. J Adolesc Health 2007;41:93-98.

18. Ho RC, Zhang MW, Tsang TY, Toh AH, Pan F, Lu Y, et al. The association between internet addiction and psychiatric co-morbidity: a meta-analysis. BMC Psychiatry 2014;14:183.

19. Young KS. Internet addiction a new clinical phenomenon and its consequences. Am Behav Sci 2004;48:402-415.

20. Park S, Hong KE, Park EJ, Ha KS, Yoo HJ. The association between problematic internet use and depression, suicidal ideation and bipolar disorder symptoms in Korean adolescents. Aust N Z J Psychiatry 2013;47: 153-159. 